\title{
Providing Quality of Service for Packet Based Services in UMTS
}

\author{
Augusto Casaca ${ }^{1}$, J. Pedro Rodrigues ${ }^{2}$, Leandro Fernandes ${ }^{2}$ \\ ${ }^{1}$ IST/INESC, R. Alves Redol, 1000-029, Lisboa, Portugal; ${ }^{2}$ OniWay, Av. Almirante Reis 65, \\ 1150-011, Lisboa, Portugal.
}

\begin{abstract}
The provision of Quality of Service support is one of the key issues in UMTS network deployment. The paper analyses the solution implemented by a UMTS network operator in its launch network. The end-to-end QoS provision problem is presented and a solution, based on specific network engineering and adaptation of the applications to the radio bearers and service classes, is introduced and analysed.
\end{abstract}

Key words: UMTS, Quality of Service, IP, ATM, Differentiated Services, Applications.

\section{INTRODUCTION}

Providing Quality of Service (QoS) in the Universal Mobile Telecommunications System (UMTS) networks is a key objective to be achieved both by manufacturers and network operators for the deployment of UMTS. The problem of QoS provision is particularly relevant for mobile packet switched based services, which constitute the main novelty introduced in UMTS networks compared to the previous generation of circuit switched wireless networks.

The structure of a UMTS network consists of two main levels: radio access network and core network. The end-to-end services are carried over the network using bearers. Bearer is a service providing QoS between two defined points. As the radio access network and core network have their own QoS properties, the QoS needs to be treated separately in each of these levels. The end-to-end QoS is the global result, which takes into account the distinct levels of the network. 
In UMTS a specific medium access control protocol is used on the radio bearers, which link the User Equipments (UE) to the base stations. From the base stations to the core network the transport of packets is done over ATM. In the core network, the information is encapsulated in IP; here, the QoS is treated according to the Differentiated Services (Diff Serv) model. The layer 2 protocol in the core network, which will transport the IP packets is not standardized, although, in practice, ATM is also one of the main choices of network operators for this purpose.

In UMTS there is one additional feature, which consists in the UEs having the ability to negotiate the QoS parameters for a radio bearer. The negotiation is always initiated by the application in the UE and the network checks whether it can provide the required resources or if rejects the request.

QoS in a network has mainly to do with the provision of resource assurance and service differentiation. Having in view the achievement of the best possible end-to-end QoS, the UMTS network has to be properly engineered, both at the radio and core levels [Dixit]. Also, special attention needs to be given to the way in which the different types of applications are adapted to the network, in particular to their use of the radio bearers and of the UMTS QoS classes defined in the standards.

This paper has the objectives of introducing the end-to-end QoS aspects in UMTS with a focus into the packet based services and also of analysing the QoS solution that a network operator will provide for the UMTS network implementation, which will be ready for operation in the last quarter of 2002. In this analysis both the engineering of the network and the adaptation of applications to the network will be considered.

The paper consists of the introduction plus five other chapters. In chapter 2 a brief review of the UMTS network architecture is done. The UMTS QoS architecture and service classes are presented in chapter 3. Chapter 4 analyses the specific network engineering used for the network implementation. Chapter 5 deals with the adaptation of the applications to the radio bearers and service classes. Finally, some conclusions are drawn in the last chapter.

\section{UMTS NETWORK ARCHITECTURE}

A simplified view of the UMTS architecture is introduced in this section so that the functionalities of the main UMTS blocks can be presented. This simplified architecture is shown in Figure 1, in which some of the internal interfaces are not shown. The UMTS network architecture has been defined by 3GPP (Third Generation Partnership Project). 3GPP has planned the evolution of the network according to a series of releases. The first one to be 
implemented is known as Release 99 and is the one considered in this paper [TS 23.002].

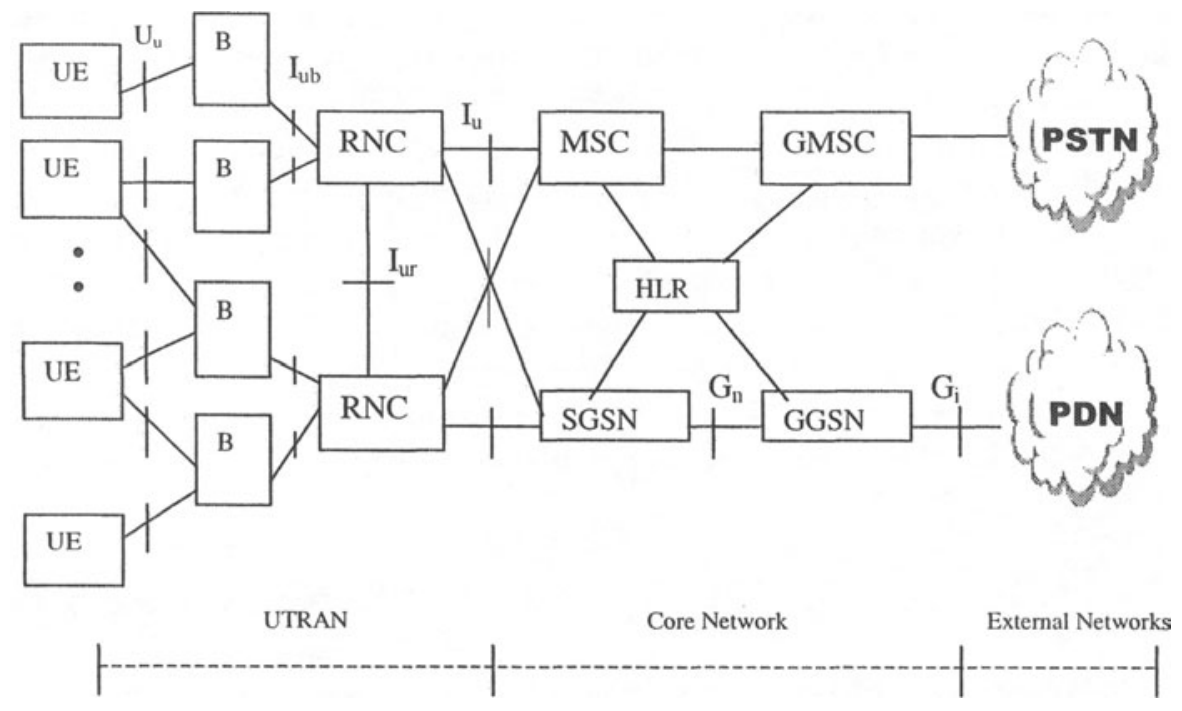

Figure 1. 3GPP Release 99 UMTS network architecture

A UMTS network consists of the radio access section, named Universal Terrestrial Radio Access Network (UTRAN), and of the core section. These two sections are separated by the Iu interface.

The UTRAN consists of a set of Base stations, known as nodes B, and a set of Radio Network Controllers (RNC). Each RNC controls a number of nodes $\mathrm{B}$. Iub is the interface between a node B and an RNC. The RNCs may communicate between themselves via the Iur interface. The radio access part comprises the network between the UEs and the nodes B (interface Uu).

The realisation of the Uu interface implies that the node B implements Wideband Code Division Multiple Access (WCDMA) radio access physical channels. The RNC is the switching and control element of the UTRAN. Each RNC is respectively connected, via the Iu interface, to the Mobile services Switching Centre (MSC) and Serving GPRS Support Node (SGSN), which are two elements of the Core Network.

The Core Network consists of a Circuit Switched (CS) domain at the upper part of the figure and a Packet Switched (PS) domain at the lower part of the figure. The main elements in the circuit switched domain are the MSC and the Gateway MSC (GMSC). The MSC is responsible for the circuit switched connection management activities including the mobility management. The GMSC takes care of the connections to other PSTN 
networks. In the packet switched section, there are also two main elements, the SGSN and the Gateway GPRS Support Node (GGSN), respectively, separated by the Gn interface. The SGSN supports packet communication towards the access network and is responsible for mobility management related issues. The GGSN maintains the connections towards other Packet Data Networks (PDN), such as the Internet, via the Gi interface.

The Home Location Register (HLR) is another main element in the core network. It contains the addressing and identity information for both the circuit and packet switched domains of the Core Network. It contains data that identifies the subscribers.

The Core Network circuit switched domain uses signalling protocols inherited from GSM. The Core Network packet switched domain can be seen as an IP backbone internal to the operator network. The Diff Serv model is used in this IP network, which operates over any layer 2 protocol.

\section{QUALITY OF SERVICE IN UMTS NETWORKS}

One of the goals of UMTS networks is to satisfy the growing demand for accessing the Internet anytime anywhere. This requires that Internet services are extended to the UE and that a QoS mechanism in the radio link is used and optimized to support Internet services. This QoS mechanism complements the one to be used in the Core Network.

A fundamental concept of UMTS is the separation of the access functionality from the Core Network functionality. The UTRAN provides access to the UEs and hides all the radio access technology dependent and mobility functions from the Core Network.

In UTRAN a transport technology is needed to interconnect the nodes B and the RNCs. The transport technology must provide differentiated QoS to multiple classes of traffic. In particular, real-time applications impose stringent delay and jitter requirements on the UTRAN transport network. In the first UMTS releases, ATM has been chosen as the transport technology in the UTRAN, because of the ATM mature schemes to support QoS.

While ATM has mature schemes to support QoS, there is also a strong interest in providing IP based transport for the UTRAN in the future. This still requires further refinements on the way that IP provides QoS guarantees, before proceeding to the field implementation. This is the path which is planned to be gradually followed from Release 4 onwards [Bos].

In UMTS Release 99, the Core Network contains a circuit switched and a packet switched domain. As a result, for an end-to-end view on QoS in Release 99, we will have to gather traditional GSM quality aspects together with IP QoS issues. There needs to be a matching of radio link bearers to the 
transport channels in the fixed part of the network. The Core Network evolution into the next releases will, however, result in a Core Network that will be packet based overall.

The UMTS QoS architecture is defined by 3GPP in the documents [TS 23.107] and [TS 23.107/4.1.0] for Release 99 and Release 4, respectively. This chapter is, however, only concerned with the QoS aspects for Release 99, as this will be the release used for the first implementations of UMTS.

The UMTS QoS architecture is shown in figure 2.

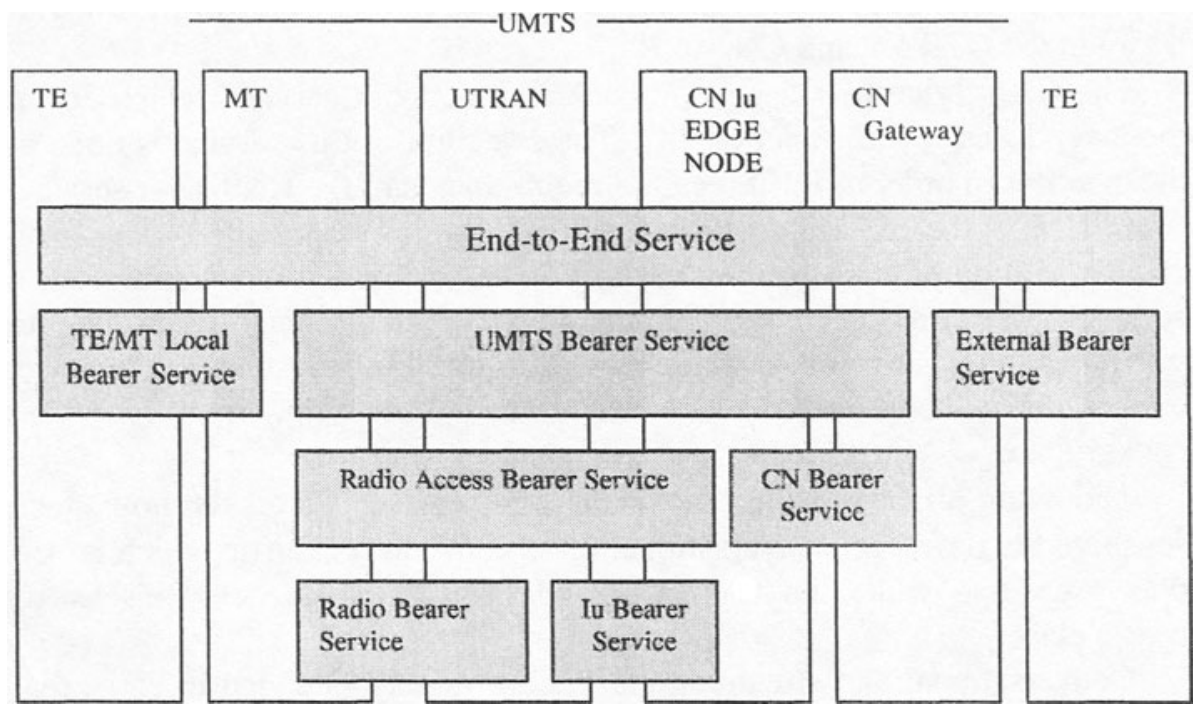

Figure 2. UMTS QoS architecture

In the UMTS QoS architecture, a bearer service defines the characteristics and functionality established between communicating endpoints to realize consistent network QoS for end-to-end services.

The end-to-end bearer service can be split into three main components, which are the Terminal Equipment/Mobile Termination (TE/MT) local bearer service, the external bearer service and the UMTS bearer service.

The TE/MT local bearer service enables communication between the different components of a mobile station. The MT is responsible for the physical connection to the UTRAN. The TE can be any terminal device, such as a laptop, a PDA or a mobile telephone. The external bearer service connects the Core Network and the destination node located in an external network. Both the TE/MT local bearer service and the External bearer service are out of the scope of this paper, as they have nothing directly to do with the network architecture. 
The UMTS bearer service uses the Radio Access Bearer (RAB) service and the Core Network $(\mathrm{CN})$ bearer service.

The $\mathrm{RAB}$ service provides transport of signalling and user data between the MT and the CN Iu edge node with the QoS established for the UMTS bearer service. This service is based on the characteristics of the UTRAN and is maintained for a moving MT. The CN service connects the UMTS CN Iu edge node with the $\mathrm{CN}$ gateway to the external network.

The RAB service is realized by a radio bearer service and an Iu bearer service. The radio bearer service is directly dependent on the UTRAN implementation. The Iu bearer service provides the transport of information between the UTRAN and $\mathrm{CN}$.

The $\mathrm{CN}$ service uses a generic backbone network service, which covers the layer 1 and layer2 functionality. The selection of this service is done by the operator in order to fulfil the QoS requirements of the $\mathrm{CN}$ bearer service.

In UMTS the provision of services assumes key importance. During the standardization process the aim was to achieve a simple classification for the services taking into account parameters such as delay, guaranteed bit rate, traffic elasticity and traffic symmetry. For this purpose four QoS service classes have been defined: conversational, streaming, interactive and background.

The main distinguishing factor between these classes is how delay sensitive the traffic is: conversational class is meant for traffic which is very delay sensitive while the background class is the most delay insensitive traffic class.

Conversational and streaming classes are mainly intended to carry realtime traffic flows. The main divider between them is traffic delay sensitivity. Conversational real-time services, like video telephony, are the most delay sensitive applications and those data streams should be carried in conversational class. Streaming services, e.g. video streaming, are usually asymmetric and allow the use of data buffering.

Interactive and background classes are mainly meant to be used by traditional Internet applications like WWW, Email and FTP. Due to less stringent delay requirements, compared to conversational and streaming classes, both provide error control by means of channel coding and retransmission. The main difference between interactive and background classes is that the interactive class is mainly used by interactive applications, such as web access, while the background class is meant for background traffic, such as e-mail delivery. 


\section{QUALITY OF SERVICE IMPLEMENTATION}

The end-to-end QoS depends on the QoS of each of the component services in the network, namely, the Local bearer service, the UMTS bearer service and the External bearer service. Altogether the end-to-end bearer service is realised by the concatenation of these three bearer services.

The Local bearer service depends on the terminal and the External bearer service depends on the service level agreement defined with the external network. As indicated before, the Local and the External bearer services are not within the scope of this paper, as they have nothing directly to do with the network architecture. Therefore we will concentrate on the UMTS bearer service. The concatenation of the Radio bearer service, Iu bearer service and Core Network bearer service forms the UMTS bearer service.

In what follows, the implementation of the UMTS bearer service, considering its three distinct components as deployed in a UMTS Release'99 network, will be analysed from the QoS provision point of view.

\subsection{Radio Bearer Service}

The Radio bearer service is defined between the UE and the RNC. In practical terms, it depends on the implementation of the Radio bearers (betweeen the UE and the node B) and of the Iub interface (between node B and RNC).

The Radio bearers carry the information between the User Terminal and the Node B. A Radio bearer is no more than a physical channel to transport information between those two points. A Radio bearer is defined by the following parameters: QoS class, uplink bit rate, downlink bit rate and type.

The standardised radio bearers for UMTS are defined in [TR 34.108]. They consist of a large number of bearers and, naturally, network operators will only deploy a restricted number of those bearers in a first phase.

In the network deployment under analysis, there will be a significant number of distinct CS and PS radio bearers at launch. This will permit a good number of options for the mapping of applications into the radio bearers. Some concrete examples of radio bearers to be deployed are:

- PS bearers, symmetrical, 64kbps (upstream)/64kbps (downstream), interactive/background;

- PS bearers, asymmetrical, 64kbps (upstream)/128kbps (downstream), interactive/background;

- PS bearers, asymmetrical, 64kbps (upstream)/384kbps (downstream), interactive/background;

- CS bearers, symmetrical, 64kbps (upstream)/64kbps (downstream);

- CS bearers, symmetrical, $12.2 \mathrm{kbps}$ (upstream)/12.2kbps (downstream). 
Besides the CS bearers, which are classical ones for the transport of voice and circuit switched data, there is a good variety of PS bearers, which allow carriage of data at different speeds. Although only the interactive and background classes are used for the PS radio bearers at the network launch, most of the streaming services can also use the interactive class. This reflects the present status of discussion in standards about the use of the streaming class, which some manufacturers consider that can be replaced by the interactive class. In a second phase of implementation the conversational radio bearers will need to be deployed to allow for the efficient carriage of bi-directional real-time services in the packet switched part of the network.

In UTRAN, Node B signalling and data are carried to the RNC over the Iub ATM interface. Node B Application Part (NBAP) protocol [NBAP] is a radio network layer protocol, which maintains control plane signalling across the Iub interface and provides a means for communication between Node B and RNC.

NBAP resides on top of the Iub transport layers and uses their services to transfer NBAP messages over the Iub interface. The transport technology used is ATM. ATM Adaptation Layer (AAL) 5 format is used for signalling and AAL2 format is used for user data. The signalling bearer for NBAP provides a reliable point-to-point connection. There may be multiple pointto-point links for NBAP signalling at the Iub interface.

\subsection{Iu Bearer Service}

The Iu bearer service is provided over the Iu interface.

The Iu interface is defined between the UTRAN and the CN. There are two instances of the Iu interface: Iucs and Iups. The Iucs interface is defined between the RNC and the MSC. The Iups interface is defined between the RNC and the SGSN. This is shown in figure 3.

In this paper we are only concerned with the provision of QoS for packet based services, therefore only the Iups interface implementation is considered.

The Iups protocol structure is shown in figure 4.

At the Radio Network Layer, the Radio Access Network Application Part (RANAP) protocol [RANAP] runs in the control plane and provides UTRAN specific signalling and control over the Iu interface. The PS domain uses the RANAP protocol to access the services provided by the UTRAN.

At the Transport Network Layer, Signalling Connection Control Part (SCCP) protocol is also used as a signalling protocol in the control plane. According to the standard, SCCP can be carried either over Broadband SS7 or over IP. For the Transport Network Layer specific transport technology, ATM is used. At the Iups interface, AAL 5 is the chosen Adaptation Layer 
to adapt GPRS Tunneling Protocol - User part (GTP-U) messages to the ATM network.

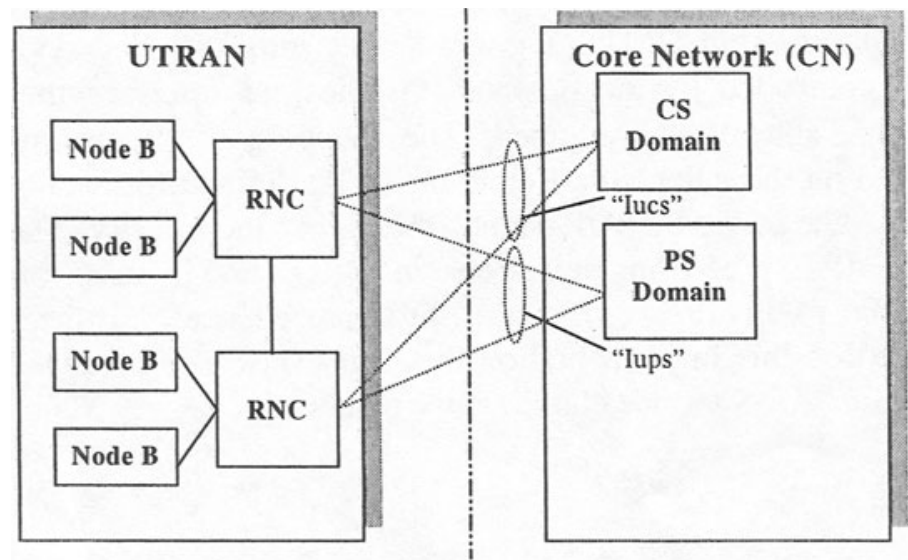

Iu Interface

Figure 3. Iu interface

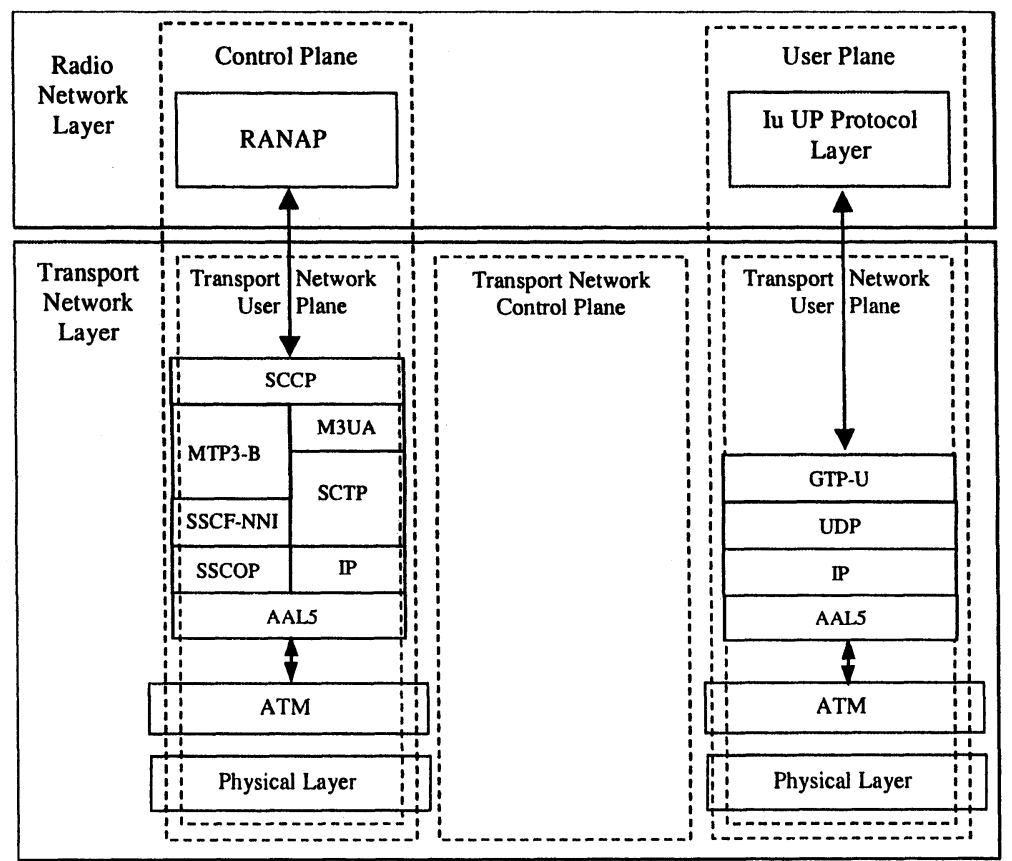

Figure 4. Iups protocol structure 
The standard defines that at the lups interface, the Diff Serv IP model defined by IETF will be used. As ATM is used as the transport bearer at this interface, the Diff Serv Per-Hop Behaviours (PHB) need to be mapped into the ATM Service Classes available in the RNC and SGSN equipments.

The mapping from UMTS service classes into Diff Serv Code Points (DSCP) is controlled by the operator. At the Iups interface the mapping indicated in Table 1 was adopted. The mapping of the service classes depends also on the Allocation/Retention (A/R) defined for each subscriber in the HLR. The lower the A/R values the higher the priority. The Assured Forwarding (AF) Per-Hop Behaviour in Diff Serv is used. In the AF notation, AFij means (i-service class; $j$-drop precedence). DE means default value. Based on this implementation the Core Network is ready to support the four distinct QoS service classes since its launch.

Table 1. Mapping of QoS service classes into DSCP

\begin{tabular}{lllll}
\hline A/R & Conversational & Streaming & Interactive & Background \\
\hline 1 & AF42 & AF31 & AF21 & DE \\
2 & AF42 & AF32 & AF22 & DE \\
3 & AF42 & AF33 & AF23 & DE \\
\hline
\end{tabular}

The DSCP mapping for Control packets at the Iups interface is indicated in table 2 .

Table 2. Mapping of control packets into DSCP

\begin{tabular}{ll}
\hline & DSCP \\
\hline Control DSCP & AF41 \\
\hline
\end{tabular}

Three types of ATM Virtual Connections (VCs) are used: Constant Bit Rate (CBR), non-real-time Variable Bit Rate (nrt-VBR) and Unspecified Bit Rate (UBR). The chosen mapping of the Diff Serv DSCP into the ATM VCs is indicated in table 3.

Table 3. Mapping of Diff Serv DSCP into ATM

\begin{tabular}{ll}
\hline DSCP & ATM VC \\
\hline AF42 & CBR \\
AF31 & Nrt-VBR \\
AF32 & Nrt-VBR \\
AF33 & Nrt-VBR \\
AF21 & Nrt-VBR \\
AF22 & Nrt-VBR \\
AF23 & Nrt-VBR \\
All Others & UBR \\
\hline
\end{tabular}




\subsection{Core Network Bearer Service}

The packet switched domain in the core network is limited by the SGSN and GGSN. The interface between these two elements is the Gn interface. The $\mathrm{Gn}$ interface protocol structure, respectively for the user and control plane, is shown in the figures 5 and 6.

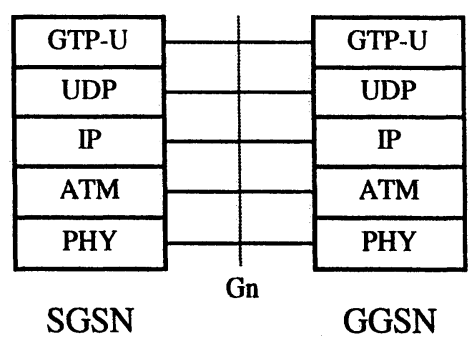

Figure 5. Gn protocol structure (user plane)

\begin{tabular}{|c|c|}
\hline GTP-C & GTP-C \\
\hline UDP & UDP \\
\hline $\mathbf{I P}$ & IP \\
\hline ATM & ATM \\
\hline PHY & PHY \\
\hline
\end{tabular}

Figure 6. Gn protocol structure (control plane)

At the Gn interface, Diff Serv will be used at the IP level. In our implementation ATM will be used as the transport bearer at this interface. This means that the Diff Serv PHBs will be mapped into the available ATM Service Classes as was indicated before. The interoperability between operators will be based on the use of Service Level Agreements at the Gi interface. The Gi interface is defined between the GGSN and External packet networks.

The mapping from UMTS service classes into Diff Serv DSCPs is controlled by the operator. The mapping of the service classes and control packets into Diff Serv DSCPs is the same as at the Iups interface. 


\subsection{QoS Negotiation}

The QoS profile subscribed by the user is stored in the HLR. In the PS domain there is a QoS negotiation among the different network elements.

A Packet Data Protocol (PDP) context is defined as an instance of a PDP type, which describes the network layer protocol used in the PS domain. It is a virtual connection between the mobile station and the GGSN. A PDP context includes an identifier, such as an IP address for sending and receiving data, and a set of characteristics, such as QoS parameter values in a QoS profile. A PDP context carries all application traffic sourced from and destined for one IP address.

After attachment, an MT requests from the SGSN a certain QoS profile for a PDP context, using an "Activate PDP context" message. The SGSN tries a best fit between the requested QoS and the one subscribed at the HLR. The result of this match is the so-called Negotiated QoS profile.

In the next phase a PDP context is created between the SGSN and the GGSN. The negotiated QoS is transferred to the GGSN, which can further negotiate it, although this does not happen in some of the first implementations, and the GGSN simply accepts the negotiated QoS profile.

Finally, a Radio bearer is allocated by the RNC. If the radio network is unable to allocate resources to satisfy the negotiated QoS requested from the SGSN, the RNC fails the assignment request, otherwise the Radio bearer is set-up and a GTP tunnel is established between the RNC and the GGSN.

\section{APPLICATIONS REQUIREMENTS}

The 3GPP standard [TS 23.107] indicates that the mapping from application attributes into UMTS service attributes is an operator's implementation issue.

In practical terms each application is considered to belong to a certain UMTS QoS class. When the Terminal requests the activation of a PDP context, it sends the QoS profile of the application as a parameter in the "Activate PDP context" message. As described in the previous section, the SGSN compares the attribute values indicated in the QoS profile requested for the application with the attribute values of the user QoS profile in the HLR and based on the negotiated QoS established by the SGSN a Radio bearer is either established or not. The DSCP value to be marked in each packet is dependent on the final QoS value agreed for the application generating the packet. The marking of packets is done at one of the entrances to the Diff Serv domain. The Diff Serv domain extends from the RNC to the GGSN, which is coincident with the existence of GTP tunnels. 
In UMTS the applications will encompass a large number of types, such as, personal communication, business applications, advertising, entertainment, image applications, travel, banking, telemetry, shopping and voice.

With respect to QoS issues many of the applications have common requirements independently of the category to which they belong. Therefore the applications can be classified into service categories, which is a classification orthogonal to the types of applications. All the applications in a service category have common QoS requirements, this means that for engineering the network the important aspect is to know to which service category the application belongs and not necessarily the type of application.

Examples of service categories are: PS based Short Message Service (SMS), Multimedia Message Service (MMS), E-mail, File Transfer, Web browsing and Streaming.

Next a table can be drawn in which each application is allocated to one or more service categories plus the respective QoS service class, degree of symmetry, end-to-end 1-way maximum delay, required data rate and tolerance to information loss. From all this information the QoS profile requested for each application can be established. The QoS profile is considered to be a single parameter with multiple data transfer attributes. The QoS profile requested for each application will be included by the Terminal as a parameter of an "Activate PDP Context Request" message, which is sent to the SGSN.

From the large number of applications to be deployed, Table 4 gives an example considering four of those applications: E-mail delivery, Directory consulting, Alerts and Sports News.

Table 4. Example of applications QoS profiles

\begin{tabular}{|c|c|c|c|c|c|c|}
\hline Application & $\begin{array}{l}\text { Service } \\
\text { Category }\end{array}$ & $\begin{array}{l}\text { QoS Service } \\
\text { Class }\end{array}$ & Symmetry & $\begin{array}{l}\text { Maximum } \\
\text { Delay }\end{array}$ & $\begin{array}{l}\text { Data } \\
\text { Rate }\end{array}$ & $\begin{array}{l}\text { Loss } \\
\text { Tolerance }\end{array}$ \\
\hline $\begin{array}{l}\text { E-mail } \\
\text { delivery }\end{array}$ & E-mail & Background & Two-way & $\begin{array}{l}10 \mathrm{~s}- \\
\text { minutes }\end{array}$ & $>32 \mathrm{kbps}$ & Intolerant \\
\hline $\begin{array}{l}\text { Directory } \\
\text { consulting }\end{array}$ & $\begin{array}{l}\text { Web } \\
\text { browsing }\end{array}$ & Interactive & $\begin{array}{l}\text { One-way } \\
\text { (Downlink) }\end{array}$ & $\sim 4 s$ & $<32 \mathrm{kbps}$ & Intolerant \\
\hline Alerts & SMS+MMS & Interactive & $\begin{array}{l}\text { One-way } \\
\text { (Downlink) }\end{array}$ & $\sim 10 \mathrm{~s}$ & $<32 \mathrm{kbps}$ & Intolerant \\
\hline Sports news & $\begin{array}{l}\text { Web } \\
\text { browsing + } \\
\text { SMS + MMS }\end{array}$ & Interactive & $\begin{array}{l}\text { One-way } \\
\text { (Downlink) }\end{array}$ & $<4 s$ & $<32 \mathrm{kbps}$ & Intolerant \\
\hline
\end{tabular}

One of the most important aspects to consider is the methodology to manage the simultaneous activation of several different applications with different QoS needs. 
Initially the network will only support one active PDP per MT. Therefore, the service category selected must correspond to the requirements of the most stringent application to be used. As a first step to solve this problem, and still for release 99 , in a phase subsequent to launch the support for PDP context modification will be implemented. This will allow the user to request a modification of the service category, without service interruption, whereas for launch the user will be obliged to tear down its PDP connection and activate a new one. The MT will send to the SGSN a "Modify PDP context Request" where the new QoS profile will be requested. The SGSN will check if the user subscribed data allows this new profile and if available network resources exist, after which it will accept or refuse the request. During the process the SGSN will have to update the GGSN via an "Update PDP context Request" and the UTRAN through an $\mathrm{RAB}$ Assignment procedure.

The definitive solution for supporting traffic flows with different QoS requirements will be available later on, when the network and user equipment will support multiple simultaneous PDP contexts with the same network address. The user or application will check the actual PDP activated and, if the profile does not fit the application needs, a new PDP context will be activated. The MT sends an "Activate Secondary PDP Context Request" message to the SGSN and this message will include a parameter which will identify the already existing PDP context. The SGSN will check if there are enough network resources for the QoS requested and, if affirmative, will create a new PDP context, which will use the same network address as the previous one.

The full description of the modification and creation of a secondary PDP context is available in [TS 23.060]. It is planned that the existing network implementation allows an evolution to the above-mentioned capabilities by the end of 2003.

Finally we come to an issue that will be central in what concerns applications. For an effective use of the network Qos capabilities the applications must be able to request and modify the required QoS profile. Although the end user may perform this manually, it is a tedious and cumbersome task. The first applications to support this will be the ones embedded in the Terminal, however, gradually, PC and PDA applications, will have to start to support the request and negotiation of QoS from the network. This will be done via the TE/MT interface and it is mainly an issue of developing software to interface the application. 


\section{CONCLUSIONS}

The provision of QoS support for packet-based services will be an important breakthrough in mobile networks. $2.5 \mathrm{G}$ networks have proved so far unable to cope with QoS support, due to radio capacity availability and development issues. With UMTS, and given the large availability of network resources at launch, mobile network operators will have the opportunity to implement QoS capabilities from day one, and define a clear roadmap for the years ahead.

With the mechanisms described the two most important requirements for packet-based services in UMTS can be met:

- Guarantee the allocation of the minimum network resources required for each type of application. Real-time packet-based services will only be successful if the probability of the service being available is very high; this means prioritizations of network resources between types of traffic with different requirements.

- Allow customer differentiation inside each class of applications. With the increasingly high penetration rates of mobile users, operators want to focus on high revenue customers, and for that, differentiation between subscriptions is crucial.

The fully standardized interfaces and protocols allow the future interworking with other mobile networks as well as with external IP networks like private Virtual Private Networks or the Internet.

\section{REFERENCES}

[Bos] L.Bos and S.Leroy, Toward an All-IP-based UMTS System Architecture, IEEE Communications Magazine, February 2001.

[Dixit] S.Dixit, Y.Guo and Z.Antoniou, Resource Management and Quality of Service in Third-Generation Wireless Networks, IEEE Communications Magazine, February 2001.

[NBAP] 3GPP TS 25.433, v3.3.0, UTRAN Iub Interface NBAP Signalling, 2000.

[RANAP] 3GPP TS 25.413, UTRAN Iu Interface RANAP Signalling, June 2001.

[Robles] T.Robles et al, QoS Support for an All-IP System beyond 3G, IEEE Communications Magazine, August 2001.

[TS 23.002] 3GPP TS 23.002 V3.4.0, Network Architecture (Release 1999), December 2000.

[TS 23.107] 3GPP TS 23.107 V3.6.0, QoS Concept and Architecture (Release 1999), April 2001.

[TS 23.107/ 4.1.0] 3GPP TS 23.107 V4.1.0, QoS Concept and Architecture (Release 4), June 2001.

[TS 23.060] 3GPP TS 23.060 V3.8.0, General Packet Radio Service;Service Description; Stage 2 (Release 99), June 2001.

[TR 34.108] 3GPP TR 34.108, Common Test Environments for User Equipment Conformance Testing, June 2001. 